\section{La importancia de la motivación económica}

Daniela Elstein *

Resumen: La motivación es considerada uno de los factores más importantes que desvela, tanto a los Administradores de las empresas, como a los responsables de Marketing.

La importancia de la motivación, no será cuestionada en las siguientes líneas, sino que dando uso a las ideas de dos grandes pensadores de la talla de Herzberg y Maslow, buscaremos una respuesta al cómo, cuándo y hasta dónde motivar a nuestro personal. Por medio de tres categorías de motivación y un anexo especial de segmentación de estas categorías, daremos respuesta a los anteriores interrogantes.

Otro punto de importancia que vamos a analizar con respecto al tema de la Motivación en los trabajadores, lo constituye el debate frecuente sobre la dicotomía representada por el homo economicus, aquél que racionaliza todas sus decisiones, en función principalmente de una ecuación costo-beneficio, versus el homo-socialis, quien tomará sus decisiones basado en su sentido de pertenencia, de reconocimiento, de poder. Este análisis, al igual que las teorías antes referidas, forma parte de la base conceptual de esta propuesta.

La idea central del presente artículo es, basándonos en el análisis de estos conceptos y valiosas teorías, establecer una Jerarquía de Necesidades y Motivaciones aplicada exclusivamente a los Recursos Humanos. Asimismo se buscará realizar una "Segmentación” del personal de las diferentes organizaciones.

Los mejores resultados no podrán lograrse con capacitaciones y beneficios aleatorios, sino estableciendo un verdadero programa de Desarrollo Estratégico del Personal Talentoso, principal activo intangible y escaso de las organizaciones de esta era. Nuestro objetivo entonces es brindar una herramienta de ayuda para lograrlo.

Palabras claves: colaboradores - desarrollo estratégico - motivadores de atracción - motivadores de pertenencia - motivadores de retención - necesidades - segmentación - talento.

[Resúmenes en inglés y portugués en las páginas 201-202]

(*) Contadora Pública Nacional (UNL). MBA Dirección Estratégica de Negocios (UB). Profesora de Marketing y de Administración en la Facultad de Diseño y Comunicación de la Universidad de Palermo. Profesora del área de Estrategia Escuela de Negocios de la UB en la Carrera MBA Dirección Estratégica de Negocios y, profesora de Costos en ONG y Costos en Empresas de Seguridad en la Especialidad de Costos del IAPUCO (Instituto Argentino de Profesores Universitarios de Costos) dirigida por el Doctor Amaro Yardin. 


\section{Introducción}

No es una novedad en el mundo empresarial, que toda organización competitiva debe estar orientada al cliente, en todas y cada una de sus actividades. Lo que hoy queremos proponer es una herramienta de importante valor para el logro de este objetivo, tan conocido y discutido en las empresas, pero pocas veces logrado. Esta herramienta, aunque con el tiempo deberá necesariamente transformarse en parte de la cultura empresaria, consiste en estar Orientados hacia los Colaboradores. No estamos dejando de lado la Orientación al Cliente sino que estamos considerando como punto de partida, como premisa fundamental, la Orientación al Colaborador, para hacer de todos, el objetivo de orientación al Cliente. Implica lograr en primera instancia una propuesta de valor para el personal de la organización, y así poder generar luego una mejor propuesta de valor al Cliente. Ambos objetivos, bien logrados, son de una sinergia valiosísima para todo tipo de empresas y organizaciones sin fines de lucro. Sabemos que los clientes al operar con la empresa son quienes permiten pagar los sueldos, abonar el consumo de energía y demás gastos de la organización. Pero quien da valor agregado a los productos y/o servicios, es el personal. Por ello es necesario y es la base de esta propuesta, instalar la política de conquistar y fidelizar clientes, pero sin dar la espalda a los Recursos Humanos; todo lo contrario, son el primer escalón para el logro de ese objetivo.

Entendemos también que el gran desafío de todo Gerente o Jefe es lograr que sus empleados hagan converger los objetivos personales o individuales con los de la empresa. ¿Pero cómo podemos lograrlo sin antes saber qué es lo que realmente motiva a nuestros colaboradores? Están siempre muy presentes en los Gerentes los objetivos empresarios, ¿pero están los de sus colaboradores?

Los reto a que entrevisten a algún Gerente, cercano a ustedes y que estén seguros que les responderá con la verdad, y no como en una evaluación anual de cumplimiento de objetivos. Traten de analizar cuánto conocen de sus empleados. La mayoría conoce muy poco, y menos aún promueve el desarrollo de sus subordinados. Quienes lo hacen, son líderes efectivos, que logran motivar a sus equipos, y obtener de ellos la mejor performance, tanto para la empresa, como para su propio desarrollo. Pero no es nuestro objetivo de hoy hablar sobre liderazgo, aún cuando está íntimamente ligado al tema que hoy nos ocupa. Motivación, Liderazgo y Comunicación, son las 3 bases del desarrollo de los Recursos Humanos, no es posible contar con un buen desempeño si alguna de las tres está en falta.

Tampoco podemos dejar de mencionar en este análisis a las Evaluaciones de Desempeño. La mayoría de las veces son catalogadas como un desperdicio de tiempo y dinero. Esfuerzo de Gerentes y Empleados para detallar objetivos que encajen en la política empresaria, y a veces, de un desarrollo de carrera teórico, casi como un concepto retórico.

Lo cierto es que el empresariado y los académicos hemos pasado mucho tiempo analizando al cliente. Lo más importante es el cliente: es cierto, no lo negamos. ¿Pero cómo sustentar una empresa orientada al cliente sin personal motivado para hacerlo? Varios autores han conceptualizado al cliente interno como parte importante de un proceso de valor para el cliente final, y sin profundizar correctamente en sus necesidades y motivaciones, en definitiva sin desarrollar el logro de la satisfacción de los clientes internos. 
A pesar de esto, cada vez más, se profundiza acerca de la importancia de conservar y desarrollar los talentos en la empresa, de la gran ventaja que significa para una organización contar con talentos, con knowledge workers. De alguna manera se va tomando conciencia de la importancia de los colaboradores, como uno de los principales activos intangibles de toda empresa.

Incluso, la forma en que llamamos a quienes prestan su trabajo y aportan sus distintas capacidades a la organización, está cambiando. El término "colaborador" que aquí usaremos indistintamente con el de empleado, personal, trabajador; es el que mejor representa la figura que entendemos deben ocupar los Recursos Humanos en las empresas. Tiene en cuenta diferentes aspectos de la persona, más allá de la simple utilización de sus competencias, considera el aspecto relacional. Son personas que dan su contribución integral, a cambio de ciertos beneficios. Sostenemos que a través de la correcta aplicación de las herramientas que ofrecemos en los siguientes párrafos, los gerentes podrán incrementar la productividad de la empresa y aumentar el bienestar general de la organización.

Antes de avanzar en el desarrollo, cabe hacernos la siguiente pregunta: ¿Qué sector de la empresa ha de ser blanco de las actividades de motivación? Hemos de responder: el ciento por ciento de la organización debe estar sumergida bajo la política de conquista, mantenimiento y motivación. Algunos detractores podrán argumentar que no tiene sentido invertir tiempo, dinero y energías en motivar al personal subalterno, o de menor jerarquía. Esto sería incurrir en un grave error, y así perderse de la ventaja que va a representar para su empresa la correcta aplicación de esta política de orientación al colaborador y herramienta de motivación. La mejor versión de una organización estará dada por aquella en donde los Recursos Humanos puedan desplegar todo su potencial, peticionar y proponer ideas que la hagan crecer, al tiempo que hacen incrementar sus necesidades de autorrealización. Con una sola idea que acallemos, estaremos silenciando la oportunidad de innovar en un mercado cada vez más cambiante y competitivo. Resumiendo: se ha analizado mucho acerca de la motivación, sobre las necesidades y el cliente interno; se ha invertido mucho tiempo y dinero en Evaluaciones de desempeño poco efectivas. Lo que proponemos aquí es sentarnos en el lugar de los colaboradores para, en primer lugar analizar qué los motiva realmente, conocerlos en profundidad, y luego ordenar y organizar esas necesidades y motivaciones las que combinadas a una segmentación del personal de cualquier organización, nos permita finalmente construir una verdadera herramienta de Desarrollo Estratégico del Personal Talentoso, y en consecuencia construir una ventaja competitiva única para la organización.

Tomando entonces como base las teorías de Maslow y Herzberg, desarrollaremos una escala de necesidades y motivadores, para nuestros colaboradores, transformándola en parte esencial de toda Evaluación de nuestros Talentos. Evaluemos no sólo su desempeño en función de los objetivos empresarios, sino también, veamos cada año, cuántas de sus expectativas fueron cumplidas. Cuáles son sus principales motivaciones para que puedan desarrollarse como personas y como profesionales en nuestra empresa.

Y califiquemos qué tipo de personal tenemos, así como analizamos a nuestros clientes. 


\section{La motivación económica}

¿Qué es más importante para la fuerza laboral: el dinero o el reconocimiento?

Si hacemos esta pregunta es probable que obtengamos respuestas variadas, aún dentro de un mismo grupo. Cada individuo, de acuerdo a sus experiencias, a su posición, a su percepción, a sus creencias, elegirá una u otra, como la más trascendente en la vida laboral de las personas.

En el siglo XVIII, cuando Adam Smith (1776) desarrolló su teoría de Homo economicus, al publicar La riqueza de las Naciones, nadie hubiera dudado en responder que lo que importa al empleado solamente es la obtención de su lucro. Repasemos rápidamente sus principales enunciados: a. Que, como ser económico, el hombre tiene el impulso natural del lucro; b. Que el universo esta ordenado de tal manera que los empeños individuales de los hombres se conjugan para componer el bien social; c. Que, conforme a. y b., el mejor programa consiste en dejar que el proceso económico siga su propio curso (laissez faire).

Asimismo concluía que el hombre adquiría una mayor aptitud para descubrir la mejor forma de hacer sus tareas, cuando tenía toda su atención en un solo objeto.

No podemos negar que en muchas organizaciones existen esta clase de trabajadores, ya sea por elección personal, por propias políticas empresarias, o con personas que han pasado por esa instancia, evolucionando luego a medida que desarrollan su carrera. Bajo estas teorías y análisis de los recursos humanos, la motivación, indudablemente, estaba concentrada en lo económico. Más tarde, a partir del desarrollo de las teorías de la Escuela de Relaciones Humanas, a principios del siglo XX, se comenzó a comprender que el hombre tiene otras necesidades además de las materiales. Se aborda el análisis del trabajador desde otra perspectiva. Se lo analiza, como ser complejo, con una orientación más psicológica. En ese momento es cuando se inician los análisis y estudios de necesidades y las motivaciones y del trabajador en tanto ser humano.

A partir del año 1925, surgen con la Escuela de las Relaciones Humanas, los conceptos de motivación, liderazgo, y comunicación. Ya no se habla solo de jerarquías o división del trabajo. La experiencia Hawthorne confirma que las recompensas económicas no son las únicas que valora el ser humano, sino que existen otras relacionadas a los aspectos sociales, al reconocimiento, al sentido de pertenencia, entre otras.

Durante el auge de esta escuela de Relaciones Humanas se relegan las necesidades materiales y se propone que la productividad y la eficiencia organizacional son siempre logradas a partir de la conformación de grupos y estrategias de motivación basadas en el trabajo en equipo.

En esa época la respuesta a nuestro interrogante hubiera sido indiscutiblemente que la motivación de los trabajadores pasa por la satisfacción de las necesidades antes mencionadas.

Varios años y teorías después, nos enfrentamos al análisis de la prioridad de una sobre la otra: ¿las económicas o las sociales? ¿Quién gana la lucha, el homo economicus o el homo socialis? ¿Qué lugar ocupa la motivación económica? ¿Desaparece como motor de motivación? Obviamente la respuesta es negativa. Lo que varía es el lugar que ocupa. Y no siempre será secundando al reconocimiento o sentimiento de pertenencia. Dependerá de la etapa de desarrollo del individuo, como persona, pero sobre todo en su carrera laboral. Eso es lo que nos interesa. Koontz (1990) define que: "las motivaciones humanas se basan en necesidades ya sean conscientes o inconscientemente expresadas...motivación: es un término genérico que se aplica a una amplia serie de impulsos, deseos necesidades, anhelos y fuerzas similares" (Koontz, 1990, p. 500-501). 
Lo que motiva al ser humano es tan amplio como lo son las necesidades, puesto que son el reflejo mismo de las necesidades o deseos.

Es por esto que se necesitan conocer las necesidades para entender qué es lo que motiva al ser humano, como individuo y en cada uno de los lugares que ocupa en su condición de trabajador, consumidor, padre, miembro de un club, etc.

La motivación económica en sí misma, es difícil de evaluar como algo objetivo. Aún cuando se traduzca en un sueldo, o un nivel de sueldos; variará en función de los puestos, la jerarquía, el esquema de retribución del rubro en el que se desenvuelva el colaborador. No es lo mismo la retribución que "espera" un vendedor, que lo que "espera" obtener un gerente de administración. Las empresas no siempre pueden satisfacer las expectativas económicas de sus colaboradores, y sin embargo estos permanecen en la organización. De ser una permanencia elegida, y no como la única opción de una fuente más o menos segura de ingresos, seguramente lo que cede en lo económico, lo recupera en otros beneficios no económicos.

Cada trabajador hace una evaluación de lo que aporta a la empresa u organización y de lo que recibe. No siempre lo que pesa más en el lado de lo que recibe es lo económico. Pero siempre estará presente como una parte importante de sus beneficios.

Concluyendo, en el desarrollo de esta herramienta, la Motivación Económica no es la única motivación de los colaboradores de una organización, pero si es una muy importante. Ocupará un lugar, que debe ser identificado, analizado y evaluado de acuerdo al "Segmento" en cuestión.

\section{Desarrollo de talentos y orientación a los colaboradores}

Como ya se dijo al comienzo de este artículo, los talentos constituyen uno de los activos más importantes, sino el más importante, de toda organización. Estos pueden llegar a representar para la compañía una ventaja competitiva difícil de imitar. Lograr el Desarrollo de esos talentos, entonces, es el puntapié inicial para obtener y mantener a lo largo del tiempo esa ventaja competitiva. El eje de este artículo es la herramienta que colaborará en la consecución de ese desarrollo, girando en torno al análisis y evaluación permanente de esos Talentos y sus motivaciones. En esto consiste lo que planteamos como Orientación a los Colaboradores.

Sabemos entonces que es importante el desarrollo de talentos, y más aún retenerlos. Pero, ¿cómo lograrlo? Muchas veces se cree que es sólo una oportunidad reservada para grandes corporaciones, que pueden destinar un abultado presupuesto a tal objetivo. Pero, realmente, esto no es así. Cada empresa debe definir el mejor Desarrollo de sus Talentos, el óptimo, de acuerdo a sus recursos: de personal y presupuestarios.

No vamos aquí a profundizar sobre el concepto de Desarrollo de Talentos, sino que vamos a desarrollar una herramienta para evaluar y analizar las necesidades y motivaciones de nuestros colaboradores, como parte esencial para su logro.

Comencemos por analizar el contexto donde se desplegará esta herramienta: empresas u organizaciones.

En un mercado con condiciones normales de oferta -sin una crisis como la que tocó vivir a nuestro país en el 2001 donde la coyuntura marcó el ritmo de las decisiones- es importante comenzar pensando cuáles son las empresas elegidas por los empleados al momento de postularse, o más aún cuáles consideran que son las mejores empresas para desarrollar sus carreras. 
En la encuesta publicada por la revista Fortune, en el año 2010, los empleados de las empresas elegidas como las mejores para trabajar, mencionan como condiciones valoradas: desde beneficios (como guardería, gimnasio, regalos), hasta la posibilidad de participar en proyectos de su elección, empleando el veinte por ciento de su tiempo regular de trabajo, es decir no mediante la utilización de horas extras. Este beneficio es otorgado por Google a sus ingenieros, empresa que desde hace varios años ocupa los primeros puestos del ranking.

Cada empresa deberá buscar cuáles son esos beneficios o motivadores que puede ofrecer a sus Talentos. Más adelante analizaremos cómo clasificar esos motivadores y cómo utilizarlos.

Por otra parte, el desafío estratégico más importante de toda empresa, es la búsqueda de una ventaja competitiva que sea difícil de imitar, que las haga únicas, que puedan permanecer en esa posición superior en el mercado y mantenerla en el tiempo. Es decir lograr una core competence, como por ejemplo Sony y la miniaturización. No es algo sencillo descubrir y explotar una ventaja como esta, requiere de mucho análisis e investigación, y también de una gran inversión. Sin embargo, hay una ventaja competitiva que está al alcance de cualquier organización. Además será siempre difícil de imitar. Esta es la ventaja que representa el staff de cada empresa. Este equipo es único e irrepetible para cada organización. Las personas son diferentes, las situaciones y su interacción, también conforman una diferencia. Ese conjunto de colaboradores, interrelacionados de manera única de acuerdo a la estructura y cultura de la empresa, puede ser una oportunidad o una amenaza. Dependerá de los objetivos y los planes de acción que establezcan quienes dirigen esas organizaciones. Las personas son únicas. Esos individuos capacitados, integrados en la cultura de la organización, como equipos y como individuos, conforman una expresión única, que puede ser aprovechada y ser una gran fortaleza para la compañía. Es una ventaja que todos pueden tener, y una oportunidad que cada empresa debería aprovechar. La mejor expresión de un equipo de trabajo será aquél que logre la automotivación, además de las motivaciones extrínsecas. Los equipos que cuenten con un buen líder, tendrán asegurada esa automotivación.

Pasemos ahora a analizar a los protagonistas de este análisis: los Recursos Humanos y sus motivaciones.

Las motivaciones de cada equipo y de cada individuo serán variadas, aún dentro de una misma empresa. Como ya lo mencionamos anteriormente, los seres humanos tienen necesidades y motivaciones diferentes.

Estos seres humanos buscarán cubrir sus necesidades básicas, pero también, como seres complejos que son, tienen otras necesidades sociales, que buscarán satisfacer en su ámbito de trabajo.

Retomando nuestra idea central, recordemos que lo que vamos a desarrollar aquí es una herramienta que nos permitirá elaborar una escala de esas necesidades. Esta herramienta se utilizará tanto para analizar y comparar el grado de cobertura de una organización, como para realizar un seguimiento y desarrollo de carrera de los empleados de una entidad.

Comencemos entonces a analizar las necesidades.

Como dijimos, el puntapié inicial lo haremos a partir de las teorías de Maslow y Herzberg. Volvamos por un momento a sus análisis.

Maslow jerarquizó a las necesidades definiendo primero las del ámbito físico, por llamarlas de algún modo: fisiológicas, las de seguridad y sociales, y, a medida que va subiendo en su escala, llega a una necesidad de autorrealización, difícil de medir, y de expresión diferente para cada ser humano. 
En el análisis que hace Herzberg, separa las necesidades entre factores de mantenimiento y motivadores. Entendiendo que los factores de mantenimiento son aquellos factores básicos que deberían existir en toda empresa que quiera subsistir en el mercado. Los motivadores serán aquellos elementos que la empresa utilizará para diferenciarse.

Lo que buscamos aquí es analizar y definir una escala de necesidades dentro del ámbito de las organizaciones, como primer paso, y en segundo lugar, utilizar esta herramienta en función de una segmentación de la plantilla de cada organización.

Esta herramienta será de utilidad también para aquellas empresas con un presupuesto limitado. Si logra segmentar su plantilla, identificando los puestos estratégicos, puede dedicar su presupuesto escaso, a beneficios otorgados a quienes tengan mayor injerencia en el logro de los objetivos estratégicos, pudiendo ser quienes generan mayores ingresos, o a quiénes son identificados como Talentos, por colaborar con el crecimiento de la compañía.

Esta escala está pensada desde el punto de vista del empleado. Por eso, el Jefe, el Gerente, el Dueño, en tarea conjunta con el Gerente de Recursos Humanos, deberá realizar permanentemente el difícil ejercicio de ponerse en el lugar de su colaborador. Deberá animarse a hacer algo que muchas veces no se hace: preguntar al empleado qué necesita.

\section{Escala de necesidades}

En primer lugar se establecen las necesidades básicas, entendiendo como tales aquellas que cualquier persona evalúa al momento de ingresar a una empresa: sueldo de convenio, obra social, espacio de trabajo, herramientas de trabajo, ambiente, entre otras, y las catalogaremos como: motivadores de atracción o ingreso.

Como ejemplo mencionemos, un obrero textil que ingresa a una compañía buscará: sueldo de convenio, buen ambiente laboral, maquinaria segura, capacitación básica y uniforme. Son necesidades básicas y de seguridad. Todo esto que mencionamos son los motivadores que harán que ingrese y cumpla con sus tareas para poder satisfacer esos deseos. En esta etapa la motivación económica, analizada previamente, ocupa un lugar importante.

Ninguna organización podrá pujar por la fuerza laboral más competitiva si no ofrece como mínimo estas condiciones, para cualquier empleado que se incorpore. Si bien, a priori, parecería algo absurdo de aclarar, no nos olvidemos que en Latinoamérica hay muchas empresas que no cumplen con estas condiciones. Incluso, hay algunas, que ocultan parte de sus colaboradores y claramente no cumplen con las condiciones mínimas de un contrato de trabajo ni de higiene. Esta situación es doblemente grave, en primera instancia porque no respetamos las condiciones básicas de trabajo y seguridad de las personas, es casi como no respetar al mismo ser humano, y además porque ya se está recayendo en la realización de una actividad ilegal. Sería un gran avance para nuestro país que un individuo al momento de incorporarse a una organización, aún cuando cuente con condiciones superiores a las básicas para ingresar a un puesto determinado, evalúe que la empresa, cumpla con estas condiciones básicas para todos sus colaboradores, formen parte de la plantilla o sean trabajadores tercerizados.

Las necesidades de crecimiento, las clasificaremos como motivadores de retención: podríamos incluir aquí los beneficios, progresos y avances de carrera, planes de capacitación, reconocimientos. Cabe aclarar que los beneficios, no siempre representan un alto costo empresario. 
Además, es mucho más costosa la fuga permanente de empleados capacitados y formados, que un día franco por el cumpleaños, y/o una orden de compra o regalo, por mencionar un beneficio de bajo presupuesto. Nuevamente aunque parece una obviedad, muchas empresas no lo traducen en acciones, aunque comparten y enuncian la teoría a la perfección.

A medida que pasa el tiempo, ya sea tiempo de trabajo en una compañía o avance en la carrera personal, todo individuo buscará motivaciones diferentes a las condiciones básicas que aceptó al ingresar o en su primer empleo. La performance de los equipos de trabajo, y de las personas individualmente, dependen de la motivación. Dependen de que tengan objetivos que puedan realizarse y compartir con la empresa.

A quienes trabajen en estas condiciones, con estas motivaciones, será más difícil tentarlos para un cambio, puesto que no sólo cuentan con las necesidades básicas satisfechas, sino que además tienen ciertos beneficios valorados por ellos. Sienten (no nos olvidemos de esto, sienten, perciben) que crecen junto con la compañía, pueden desarrollar su carrera y desarrollarse como personas dentro de la organización.

Se acostumbran a la cultura, y se sienten protegidos. Más allá de lo económico, una persona que encuentra un lugar donde es valorado y reconocido; donde puede presentar una propuesta o proyecto y es escuchado y valorado; es muy factible que lo económico no juegue un papel trascendental, siempre que se encuentre en un rango razonable dentro de los parámetros del mercado. No lo olvidemos, hay que escuchar a los colaboradores, de una u otra forma, escuchar y analizar lo que tienen para decir.

Así como a los clientes que perciben cubiertas sus expectativas con el desempeño de la compañía se los denomina clientes satisfechos, y son clientes muy valorados por cualquier empresa; también existen los empleados satisfechos. Y son los empleados que se encuentran bajo este rango o categoría.

Las necesidades de autorrealización, serán aquellas necesidades que están por encima de las expectativas de todo colaborador, y que harán que califique a su organización como la mejor en el mundo para trabajar, se tendrá así un empleado leal, fidelizado. Estos serán los Motivadores de Pertenencia. Hay que seleccionar a quiénes fidelizar. ¿¿Quiénes serán estos empleados? No necesariamente los de mayor jerarquía. Es importante definir categorías, y hacer selecciones, y aquí es donde adquiere una gran preponderancia la segmentación que expondremos a continuación. Dentro de la organización existirán empleados que no estarán en este nivel, pero si se tiene empleados leales, en diferentes sectores, jerarquías o equipos de trabajo, se asegura un buen clima de trabajo y una alta probabilidad de contar con un equipo automotivado. La performance y la productividad de estos empleados, al contar con un alto grado de pertenencia, será inmejorable. ¿Cuáles pueden ser estos motivadores? No existe una respuesta universal ni una receta mágica. Tampoco el decálogo de motivadores. Este decálogo, en todo caso, será redactado por cada empresa, de acuerdo al rubro, al presupuesto de la misma, entre otros condicionantes. Repetimos el ejemplo de Google, a sus ingenieros les permite participar en los proyectos que elijan. Una pequeña empresa podría organizar un concurso de proyectos de innovación, con un presupuesto asignado. Para cada empresa la realidad será diferente, en función de los beneficios o motivadores que son considerados como "habituales" por los empleados. Si es algo habitual el beneficio del gimnasio, no será un motivador de pertenencia, será uno de retención. 
Debemos destacar además que los Motivadores pueden variar en un período de tiempo. Cada compañía deberá establecer sus 3 clases de motivadores para un lapso definido. Deberá revisarlo permanentemente para controlar que lo planificado esté cumpliéndose y generando los objetivos planeados.

Habrán dos controles: uno de seguimiento, para evaluar los resultados y otro al finalizar el período establecido (entre 3 y 5 años) para analizar nuevamente la situación motivacional en la compañía y modificar, en el caso de ser necesario, las categorías de motivadores.

\section{Segmentación}

Una de las definiciones de segmentación de mercado, enunciada por Santesmases Mestre, Sánchez de Dussso y Kosiak de Gesualdo (2000), en Marketing: Conceptos y Estrategias, dice que:

La segmentación es un proceso de división del mercado en subgrupos homogéneos, con el fin de llevar a cabo una estrategia comercial diferenciada para cada uno de ellos, que permita satisfacer de forma más efectiva sus necesidades y alcanzar los objetivos comerciales de la empresa. (Santesmases Mestre, Sánchez de Dussso y Kosiak de Gesualdo, 2000, p. 213)

Aquí proponemos hacer una diferenciación o división del personal de una compañía con una mirada más profunda en los colaboradores de las organizaciones. En varias ocasiones las divisiones del personal tienen que ver con objetivos funcionales, o de unidades de negocios, o de escalas salariales que son de interés para la administración de la empresa. Aquí buscamos dividir al personal, de modo que nos permita utilizar la herramienta de los motivadores. Podremos así calificar cuál es la categoría actual de motivadores presentes en tal o cual segmento y planificar la estrategia de avance para cada uno. Tengamos en cuenta que cada segmento (y cada individuo también) pasará por, al menos, las dos primeras categorías.

El proceso permitirá, sobre todo a las empresas con gran cantidad de empleados, planificar y establecer un Desarrollo Estratégico de sus Talentos puntual para cada grupo. No sólo los altos ejecutivos representan un talento para la empresa. También puede ser un operario, altamente calificado para sus funciones y con un gran potencial y capacidad de liderazgo.

La segmentación puede realizarse de acuerdo a los siguientes parámetros:

- Por puestos estratégicos: Los puestos estratégicos son aquellos que son de especial importancia para el desarrollo de la estrategia de la empresa. Pueden existir a diferentes niveles, operativos o de Management. La empresa podrá segmentar estos puestos de acuerdo a diferentes grados de relación con el logro estratégico. Se denominan entonces Puestos Estratégicos de Primer grado, de Segundo grado y así sucesivamente. Por razones obvias de practicidad, serían entre 2 y hasta 5 segmentos.

- Por Jerarquías: Esta división es más fácil puesto que coincide con el organigrama de la organización. Los segmentos podrían ser: Operativos, Mandos Medios, Gerentes. Esta división, si bien es sencilla y apta para todo tipo de empresas, tiene como desventaja el riesgo de estar motivan- 
do a la persona equivocada, sólo por pertenecer a una jerarquía diferente, o dejando de motivar a un potencial talento. Sería una especie de riesgo por generalización. Por eso le recordamos que aunque Segmente en función del Organigrama, debe motivar a todos.

- Por Antigüedad: Esta división es también un sistema simple y aparentemente justo. A mayor antigüedad mayor Motivación. El punto aquí es que muchas veces los empleados más antiguos son los menos motivados y menos susceptibles de serlo. Deberían establecerse segmentos como por ejemplo: 0-5 años de antigüedad; 5-10 años de antigüedad; más de 10 años.

- Por logro de objetivos: esta segmentación podría hacerse en toda empresa que tenga evaluación de desempeño bien realizada, o que aplique el sistema de Administración por Objetivos. Es complicado realizar la segmentación en función del porcentaje de cumplimiento, pero es, desde luego, la más estimulante. Casi como integrando un círculo virtuoso con los motivadores. El riesgo aquí es no prestar atención a quien no cumple con los objetivos por no estar correctamente motivado, o en un puesto de trabajo erróneo, en cuyo caso será muy difícil que se revierta su situación. Estará integrando un círculo vicioso.

También existe la posibilidad de realizar un mix, combinando dos de ellas, que debería organizar la empresa, en función de sus posibilidades y estructura.

Una vez que se haya segmentado a la plantilla, se deberá comenzar a trabajar con el Plan de Desarrollo Estratégico de Talentos. ¿Cómo? Conformando el grupo de acciones que integrarán cada etapa de Motivación. Una vez más, previo al Plan de Desarrollo, sugerimos la realización de una encuesta formal o informal entre los empleados. Para cada Segmento, se definirá en qué etapa de Motivación la empresa juzga que debe estar el mismo, y establecerá un Plan de Desarrollo Estratégico, alineado a la Estrategia de cada organización.

\section{Reflexiones finales}

Lejos han quedado ya aquellos Tiempos Modernos donde el hombre era considerado, en el ámbito de las empresas, casi como parte de la maquinaria. Se retribuía por su trabajo rigurosamente esquematizado, porque se entendía que sólo eso buscaba: su lucro.

Con el correr del tiempo y la evolución de las teorías de Administración, se comienza a considerar y evaluar al hombre como ser social. Se comprende que estableciendo condiciones óptimas de trabajo se puede lograr una mejor performance.

En el mundo actual, veloz y turbulento, donde casi no hay tiempo para el análisis, nos vamos dando cuenta que lo más importante de las organizaciones son las personas, y por eso hay que cuidarlas y retenerlas. Es la era del Conocimiento, y éste está íntimamente ligado a las personas. Hay que incluir al personal dentro de la estrategia. Es esencial contar con talentos para su desarrollo. Y por lo tanto la base está en el Desarrollo Estratégico de los Talentos.

Nada mejor para el desarrollo del personal que apuntar a su motivación. Sin embargo, entendemos que la motivación, uno de los tres pilares más importante del mejor desempeño de los recursos humanos es, cuanto menos, complicada. Los seres humanos son complejos, y por 
eso, sus motivaciones son variadas. Dependerá de su formación, del puesto que ocupe, de su percepción de la realidad.

Es importante entender que así como la motivación es uno de los pilares para el desarrollo de la mejor performance de los Recursos Humanos, la propuesta aquí planteada entiende que el pilar de competitividad de cada empresa o institución estará representado en la Orientación hacia el Colaborador, para luego lograr la Orientación al Cliente.

Luego de las sucesivas crisis del capitalismo, sobre todo a partir de la última crisis del 2008, con uno de los mayores exponentes en la quiebra de uno de los bancos más grandes de USA, el Lehman Brothers, hay un intenso cuestionamiento al capitalismo como sistema. El capitalismo en sí mismo no es un monstruo cruel y despiadado, el problema son aquellos que hacen abuso del sistema y se olvidan de los valores que deben conducir su vida, no sólo como padres o amigos, sino también como responsables de una organización, como buenos capitalistas, aunque por el momento esto resulte un oxímoron.

Cuidar y respetar a las personas que integran una organización, no sólo es una responsabilidad de los empresarios, sino que además éstos deben entender que es la mejor forma de competir en el sistema actual, desarrollando el personal y principalmente el personal estratégico. Entender que los empleados, y todos los colaboradores, son socios en ese sistema u organización, de una u otra manera, aún cuando no posean acciones de la compañía.

El empresario debe comprender la importancia de contar con personal fidelizado y leal o, como mínimo, satisfecho. Ésta será la mejor forma de lograr sus objetivos estratégicos y un buen posicionamiento en el mercado, sostenido por la ventaja competitiva única de su equipo de trabajo con un desarrollo óptimo. Esta es la base del tan mentado Desarrollo Sustentable, de toda organización.

Si al personal no se lo motiva, y sus expectativas están muy por encima de los beneficios y motivaciones cubiertas por la organización, nos enfrentamos con un serio peligro que existe en una empresa: personal insatisfecho. No sólo porque no cumple con los objetivos mínimos, sino que además, tiene la capacidad de erosionar la empresa. Peor aún será la situación de aquélla empresa que advierta, muy tarde, que posee personal insatisfecho. Es difícil, pero puede suceder, si los Directores están muy lejos del personal, y quienes deben mantenerlos informados, tergiversan o no entregan la información correcta.

La figura del personal insatisfecho también aparece en la fuerza laboral disponible en el mercado. La empresa podrá encontrarse con ellos al momento de demandar personal nuevo: cuando las organizaciones necesitan contratar personal para puestos vacantes o incremento de la plantilla. El personal insatisfecho es aquél que por motivos de coyuntura o personales no consigue un puesto de trabajo acorde a sus expectativas; también incluimos a aquél trabajador que se postula a un puesto pero no posee la capacidad ni la formación mínima requerida. A este personal lo denominaremos "Personal Residual".

El personal más capacitado y en mejores condiciones ofrecerá sus servicios a aquéllas organizaciones que estén dispuestas a cubrir sus expectativas y fomentar su progreso. Si la empresa no ofrece condiciones básicas exigidas en el mercado laboral, seguramente sólo podrá contratar "Personal Residual", que aceptarán estas condiciones inferiores.

Si la organización consigue cubrir las expectativas de los empleados, tendrá una ventaja representada por el personal satisfecho, motivado, que como grupo desempeñará las funciones con 
una alta performance. Además buscará generar mejoras, propondrá ideas, que pueden ayudar al logro de los objetivos estratégicos de la compañía.

Contar con personal fidelizado, genera además una poderosa energía dentro de la compañía. Favorece tanto al trabajo en sí mismo, como al ambiente laboral. Hace que la empresa sea atractiva para los talentos del mercado. Contagia su espíritu emprendedor y automotivante al resto de la compañía. Es el mejor elemento de inducción para un colaborador que ingresa.

La herramienta que aquí proponemos, partiendo de una Orientación al Colaborador, brinda la ayuda necesaria para la organización, comenzando con la realización de una "Segmentación", en función del tipo de empresa y plantel, y luego desarrollando un Programa ordenado de Motivación.

Si la empresa cuenta con personal satisfecho es muy probable que podamos tener clientes satisfechos. Sin embargo, si se tienen clientes satisfechos y personal insatisfecho, en el corto o mediano plazo se comenzarán a perder esos tipos de clientes. Lo más probable además es que este personal insatisfecho genere la fuga de la empresa del personal mejor capacitado, y el ingreso del personal residual.

Entonces, si buscamos lo mejor para la organización, si queremos lograr una Ventaja Competitiva Única, brindada por la existencia de una estructura de personal óptima y motivada, el consejo es:

- Desarrolle la filosofía de Orientación al Colaborador.

- Segmente su personal.

- Establezca un plan de Desarrollo Estratégico de su Talento, sobre la base de las 3 categorías de Motivadores.

Las ideas antes desarrolladas no son meramente para efectuar un análisis del estatus de la organización y su personal, sino que son de aplicación directa y concreta en consideración a los tiempos que corren; donde ya no basta con producir más, mejor y a menores costos, donde el éxito lo tendrán quiénes consideren al conocimiento un activo estratégico. Estamos inmersos en un mercado que nos desafía constantemente a reinventarnos. Las viejas recetas empresariales que alguna vez aseguraron el éxito ya no son efectivas. Las nuevas reglas impuestas por los "consumidores informados", a raíz de la revolución tecnológica, nos fuerzan a innovar o perecer en el letargo de la inacción.

Una vez que asimilemos que el camino al éxito en la nueva era está dado por el conocimiento y la innovación, buscaremos realmente las formas de hacer proliferar la creatividad y dinamismo en los únicos activos irrepetibles que posee nuestra organización: los colaboradores.

Por medio de una estructura organizacional que no sofoque la iniciativa personal y una motivación bien lograda, utilizando las herramientas aquí analizadas, obtendremos cambios importantes que nos redituarán en la consecución de ventajas competitivas sostenibles y, por ende, en el logro de un mejor posicionamiento. Y lo que es más importante, lo haremos contando con personal fidelizado, que se sentirá valorado y apreciado por su empresa, que se ocupará permanentemente de su desarrollo, alineando sus metas personales con los objetivos organizacionales. Una vez más, hagamos de nuestra cultura empresaria la Orientación al Colaborador, para llegar a nuestros clientes. Tengamos colaboradores satisfechos y ellos nos asegurarán la satisfacción de nuestros clientes. 


\section{Referencias Bibliográficas}

Koontz H. y Weirich H. (1990). Administración (9a Ed.) México: Mc Graw Hill Interamericana. Leadership, Peter Drucker, Artículo sobre entrevista con Patricia Galagan, Training \& Development, Septiembre 1998, 52, 9 ABI/INFORM Global Página 22

Smith, A. (1776/2004). La riqueza de las naciones. Colección: Clásicos de siempre. Editorial Longseller.

Santesmases Mestre, M., Sánchez de Dusso, F. y Kosiak de Gesualdo, G. (2004) Marketing: Conceptos y Estrategias. (2a Ed.) Madrid: Pirámide.

“The Best companies to work", Revista Fortune. Disponible en: http://money.cnn.com/magazi nes/fortune/bestcompanies/2011/snapshots/1.html

Summary: Motivation is considered one of the most important factors both for the directors of companies, such as those responsible for Marketing.

The importance of motivation will not be questioned in the following lines, but making use of the ideas of two great thinkers of the stature of Herzberg and Maslow, seek an answer to how, when and to what motivate our staff. Using three categories of motivation and a special segmentation of these categories, we will answer the above questions.

Another important point we will discuss it is the frequent discussion on the dichotomy represented by homo economicus, the one that streamlines all its decisions based primarily on a cost-benefit equation, versus homo - socialist, who will make their decisions based on their sense of belonging, recognition and power. This analysis, like the aforementioned theories, is part of the conceptual basis of this proposal.

The central idea of this article is based on the analysis of these concepts and theories, to establish a hierarchy of needs and motivations applied only to Human Resources. Also will try to hold a "segmentation" of personnel from different organizations.

The best results can not be achieved through training and benefits, but by establishing a genuine program of Talented Staff Strategic Development, the leading intangible asset in present organizations. Our goal then is to provide a tool to help to achieve this.

Keywords: employees - motivating attraction - motivational belonging - needs - retention motivators - segmentation - strategic development - talent.

Resumo: A motivação é considerada um dos fatores mais importantes que preocupam aos administradores das empresas e aos responsáveis de marketing.

Este artigo procura dar resposta a como, quando e até onde motivar aos empregados das empresas, segundo as idéias de Herzberg e Maslow. Através de três categorias de motivação e um anexo especial de segmentação de estas categorias, se procurará dar resposta a estes interrogantes.

Outro ponto importante que analisa o artigo -em relação à motivação- é o debate sobre a dicotomia representada pelo homo economicus, aquele que racionaliza todas suas decisões, em função da equação custo-benefício, versus o homo-socialis, que tomará suas decisões baseado no seu sentido de pertencia, de reconhecimento, de poder. Esta análise ao igual que as teorias 
antes referidas, forma parte da base conceitual desta proposta.

A idéia central do artigo é estabelecer uma Hierarquia de Necessidades e Motivações aplicada exclusivamente aos Recursos Humanos. Também se procurará realizar uma Segmentação dos empregados das diferentes organizações.

Os melhores resultados não poderão lograr-se com capacitações e benefícios aleatórios, senão estabelecendo um verdadeiro programa de Desenvolvimento Estratégico do Pessoal Talentoso, principal ativo intangível e escaso das organizações desta era. Nosso objetivo é brindar, então, uma ferramenta de ajuda para lográ-lo.

Palavras chave: colaboradores - motivadores de atração - motivadores de retenção - motivadores de pertencia - necessidades - desenvolvimento estratégico - segmentação - talento. 